\section{Topiramate and asymptomatic ocular angle narrowing: a prospective pilot study}

DYL Leung ${ }^{1}, \mathrm{H}$ Leung $^{2}$, N Baig ${ }^{1}, \mathrm{P} \mathrm{Kwan}^{2}$,
YYY Kwong ${ }^{1}$, KS Wong ${ }^{2}$ and DSC Lam ${ }^{1}$

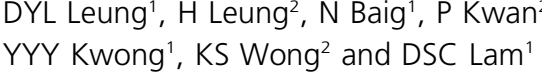

\begin{abstract}
Purpose To investigate whether subclinical ciliochoroidal effusion and resulting asymptomatic angle narrowing occurs in patients taking topiramate, by ultrasound biomicroscopy (UBM).

Methods Chinese patients aged 18-75 years for whom topiramate was indicated were recruited. Examinations including UBM were performed before and 4 weeks after commencement of topiramate.

Results In this pilot of 20 eyes of 20 patients, there were no statistically significant changes in the angle parameters noted on gonioscopy or UBM, including anterior chamber depth, angle-opening distance at $500 \mu \mathrm{m}$, trabecular ciliary process distance, trabecular-iris angle, and scleral thickness.

Conclusion Short-term use of topiramate does not induce asymptomatic angle narrowing.
\end{abstract}

Eye (2009) 23, 2079-2081; doi:10.1038/eye.2009.93; published online 1 May 2009

Keywords: Topiramate; acute angle-closure glaucoma; ultrasound biomicroscopy

\section{Introduction}

There were reports of acute angle closure (AAC) associated with topiramate use, ${ }^{1-3}$ which may involve ciliochoroidal effusion, forward displacement of iris-lens diaphragm, and secondary angle closure. The pathophysiology is as yet unclear but is presumed to be related to the sulfonamide moiety of topiramate. Adverse reactions to sulfonamides encompass a spectrum of diseases from immune reactions (Gell-Coombs Types I-IV) to idiosyncratic reactions (serum-like sickness and other hypersensitivity syndrome). ${ }^{4}$ The risk of symptomatic adverse reaction to sulfonamide was reported to be $3 \% .^{5}$ Although symptomatic AAC drives patients to seek care and hence are detected in our adverse reaction reporting system, it is unknown whether subclinical effusions with resulting asymptomatic angle narrowing occurs in patients taking topiramate. Asymptomatic angle closure may be associated with elevated intraocular pressure (IOP) and if the closure becomes chronic for regular topiramate use, chronic angle closure glaucoma (CACG) may result, which will be symptomatic only with advanced glaucomatous optic nerve damage or when an additional attack of AAC occurs. This is of relevance, particularly to Asian Chinese as they are known to have a high prevalence of pre-existing narrow angle. ${ }^{6-9} \mathrm{We}$ investigated in this pilot study, whether asymptomatic angle narrowing occurs in Chinese patients taking topiramate using ultrasound biomicroscopy (UBM).

\section{Materials and methods}

The study was approved by an institutional ethics committee. Consecutive patients of Chinese ethnicity aged $18-75$ years, for whom topiramate was indicated (migraine prophylaxis/ refractory epilepsy), were recruited from a university neurology clinic in 2007. Patients with recent exposure to topiramate ( $<3$ months), moderate-to-severe renal failure, renal calculi, hepatic failure, known AAC or CACG, were excluded. Pregnant women or women of child-bearing age not practising an acceptable means of contraception were excluded. Written informed consent was provided by all subjects. We certify that all applicable institutional and governmental regulations concerning the ethical
${ }^{1}$ Department of Ophthalmology and Visual Sciences, The Chinese University of Hong Kong, Hong Kong Eye Hospital, Kowloon, Hong Kong

${ }^{2}$ Department of Medicine and Therapeutics, The Chinese University of Hong Kong, Prince of Wales Hospital, Hong Kong

Correspondence: DYL Leung,

Department of

Ophthalmology and Visual Sciences,

The Chinese University of Hong Kong,

2/F., Hong Kong Eye

Hospital,

147K Argyle Street, Kowloon,

Hong Kong,

Hong Kong

Tel: + 8522762 3000;

Fax: + 85227687058

E-mail: dexleung@

alumni.cuhk.net

Received: 30 October 2008 Accepted in revised form: 21 March 2009 Published online: 1 May 2009

Proprietary interest: None

Conflict of interest: None 
use of human volunteers were followed during this research.

Before commencement of topiramate, each patient was examined using a standard protocol, including bestcorrected visual acuity (BCVA), refraction, Goldmann applanation tonometry (GAT) (median of five IOP measurements with time noted), dark-room gonioscopy with indentation and Shaffer's grading, and vertical cupto-disc ratio (VCDR). UBM was then performed under standardized conditions at scotopic lighting with 0.23lux intensity and a small-sized cup. ${ }^{10}$ Data collected at 12:00 a.m. were not used but the mean of three measurements at 03:00, 06:00 and 09:00 p.m. was taken to avoid falsely opened angle by the cup. ${ }^{10}$ Accommodation was standardized by looking at a target in the ceiling. Parameters derived from UBM included anterior chamber depth (ACD), angle-opening-distance at $500 \mu \mathrm{m}$ from the scleral spur (AOD500), trabecular iris angle (TIA), trabecular ciliary -process distance (TCPD), and scleral thickness at 06:00 p.m. $4 \mathrm{~mm}$ posterior to the scleral spur. Finally, a dilated fundal examination was conducted.

After baseline measurements, topiramate was commenced at $25 \mathrm{mg}$ daily through a titration schedule over 4 weeks (with a weekly increment of $25 \mathrm{mg}$ ) to a therapeutic dose of $50 \mathrm{mg}$ twice daily. Ophthalmic examinations (including UBM) were repeated at the end of week 4 . Compliance was checked by pill count.

Data were analyzed using the Statistical Package for Social Sciences (SPSS) program for Windows version 13.0 (SPSS Inc., Chicago, IL, USA). The ocular parameters of the right eyes before/after treatment were compared using Wilcoxon signed-rank test. A $P$-value of $<0.05$ was defined as statistically significant. Only patients who underwent two ophthalmic examinations and completed the dosing schedule had their data analyzed. Drawing from published data, ${ }^{11}$ in which a cut-off value of AOD500 at $\leqslant 102 \pm 84 \mu \mathrm{m}$ for gonioscopically narrow angle (Shaffer grade 0-1) and a cut-off value of AOD500 at $>291 \pm 61 \mu \mathrm{m}$ for a gonioscopically open angle (Shaffer grade 3-4) were used, ${ }^{11}$ a sample size of 20 will give a power $>90 \%$ at $P=0.05$.

\section{Results}

Twenty-three patients received two ophthalmic examinations. Data from three patients who did not complete the dosing schedule were excluded for analysis. Of the 20 patients included ( 3 males, 17 females, mean age 37.8 years, range $22-57$ years), topiramate was given for migraine prophylaxis in 16 and for refractory epilepsy in 4 patients.

There were no statistically significant changes over the 4-week treatment period in BCVA, IOP, or VCDR (Table 1). No significant changes in the angle parameters were noted on gonioscopy or UBM, as shown in the AOD500 and TIA. There were no significant changes in the position of the ciliary body relative to the scleral spur, as shown by the TCPD, refractive changes, or forward movement of lens-iris diaphragm as per ACD. We did not detect any ciliochoroidal effusion or significant changes in scleral thickness. No patient developed angle narrowing or angle closure. Despite the lower prevalence of narrow angles at a younger age, two patients met the

Table 1 Ocular parameters of the subjects $(n=20)$

\begin{tabular}{|c|c|c|c|}
\hline Ocular parameters & $\begin{array}{l}\text { Values at baseline } \\
\quad(\text { mean } \pm S D)\end{array}$ & $\begin{array}{l}\text { Values after } 4 \text { weeks' topiramate } \\
\text { treatment (mean } \pm S D)\end{array}$ & $\begin{array}{l}\text { P-value (Wilcoxon signed- } \\
\text { rank test) }\end{array}$ \\
\hline Best corrected visual acuity (Snellen) & $0.95 \pm 0.15$ & $0.97 \pm 0.16$ & 0.522 \\
\hline Spherical equivalent (Dioptre) & $-2.59 \pm 4.23$ & $-2.55 \pm 4.44$ & 0.073 \\
\hline IOP (mm Hg) & $14.5 \pm 2.6$ & $13.5 \pm 1.8$ & 0.394 \\
\hline VCDR & $0.38 \pm 0.11$ & $0.38 \pm 0.10$ & 0.999 \\
\hline Superior angle grade (Shaffer) & $2.75 \pm 0.91$ & $2.60 \pm 0.99$ & 0.461 \\
\hline Temporal angle grade (Shaffer) & $2.90 \pm 0.91$ & $2.75 \pm 0.97$ & 0.480 \\
\hline Inferior angle grade (Shaffer) & $2.95 \pm 0.69$ & $2.85 \pm 0.75$ & 0.680 \\
\hline Nasal angle grade (Shaffer) & $2.65 \pm 0.88$ & $2.65 \pm 0.88$ & 1.000 \\
\hline $\operatorname{ACD}(\mathrm{mm})$ & $2.66 \pm 0.36$ & $2.65 \pm 0.34$ & 0.765 \\
\hline AOD $500(\mu \mathrm{m})$ & $367.1 \pm 140.8$ & $385.0 \pm 150.6$ & 0.263 \\
\hline TCPD $(\mu \mathrm{m})$ & $1082.3 \pm 153.8$ & $1077.8 \pm 208.0$ & 0.985 \\
\hline TIA (degree) & $29.3 \pm 7.0$ & $30.1 \pm 9.3$ & 0.263 \\
\hline $\begin{array}{l}\text { Scleral Thickness at } 6 \text { o-clock, } 4 \mathrm{~mm} \text { posterior to } \\
\text { scleral spur }(\mu \mathrm{m})\end{array}$ & $1043.1 \pm 110.9$ & $1035.1 \pm 132.2$ & 0.638 \\
\hline
\end{tabular}

$\mathrm{ACD}=$ anterior chamber depth; AOD 500=angle opening distance at $500 \mu \mathrm{m}$ from scleral spur; IOP=intraocular pressure; $\mathrm{SD}=\mathrm{standard}$ deviation; $\mathrm{TCPD}=$ trabecular ciliary process distance; TIA=trabecular-iris angle; VCDR=vertical cup-to-disc ratio.

Note: The gonioscopy grading in dark-room test was reported here. All ultrasound biomicroscopy parameters represent the mean of three measurements from 3, 6 and 9 o'clock. 
criteria for pre-existing narrow angle (baseline mean AOD500 at 209 and $293 \mu \mathrm{m}$; mean TIA at $11.4^{\circ}$ and $14.8^{\circ}$ respectively). No significant changes in ocular parameters were noted in these two patients after topiramate use.

\section{Discussion}

Our pilot study suggests that short-term use of topiramate does not induce asymptomatic angle narrowing. Topiramate is a sulfamate-substituted monosaccharide and its role in causing AAC may well be an idiosyncratic one. A 4-week period is a reasonable time for detecting topiramate-induced ocular complications because earlier studies showed that the mean time to onset was 10 days after topiramate use at a dose as low as $50 \mathrm{mg}$ daily. ${ }^{1}$

We did not include patients with known AAC or CACG on ethical grounds. However, two of our patients met the criteria for a pre-existing narrow angle and no change in angle status was noted after topiramate use. There was not enough evidence to suggest that a preexisting narrow angle is a contraindication for topiramate use. We acknowledged the small sample size of this study. Further long-term studies of patients with a pre-existing narrow angle are warranted to delineate the ocular safety of topiramate in these patients.

\section{Acknowledgements}

The authors thank the Neurology team, The Chinese University of Hong Kong, at the Prince of Wales Hospital, Shatin, NT, Hong Kong, and Dr Felix Li at the Hong Kong Eye Hospital, Kowloon, Hong Kong, for their assistance in various stages of the project.

\section{References}

1 Fraunfelder FW, Fraunfelder FT, Keates EU. Topiramateassociated acute, bilateral, secondary angle-closure glaucoma. Ophthalmology 2004; 111(1): 109-111.

2 Boentert M, Aretz H, Ludemann P. Acute myopia and angle-closure glaucoma induced by topiramate. Neurology 2003; 61(9): 1306.

3 Levy J, Yaqev R, Petrova A, Lifshitz T. Topiramate-induced bilateral angle-closure glaucoma. Can J Ophthalmol 2006; 41(2): 221-225.

4 Tilles SA. Practical issues in the management of hypersensitivity reactions: sulfonamides. South Med J 2001; 94: 817-824.

5 Panday VA, Rhee DJ. Review of sulfonamide-induced acute myopia and acute bilateral angle-closure glaucoma. Compr Ophthalmol Update 2007; 8(5): 271-276.

6 Lam DS, Tham CC, Lai JS, Leung DY. Current approaches to the management of acute primary angle closure. Curr Opin Ophthalmol 2007; 18(2): 146-151.

7 Lam DS, Leung DY, Tham CC, Li FC, Kwong YY, Chiu TY et al. Randomized trial of early phacoemulsification vs peripheral iridotomy to prevent intraocular pressure rise after acute primary angle closure. Ophthalmology 2008; 115(7): 1134-1140.

8 Tham CC, Kwong YY, Leung DY, Lam SW, Li FC, Chiu TY et al. Phacoemulsification vs combined phacotrabeculectomy in medically controlled chronic angle closure glaucoma with cataract. Ophthalmology 2008; 115(12): 2167-2173.

9 Bonomi L, Marchini G, Marraffa M, Bernardi P, De Franco I, Perfetti $S$ et al. Epidemiology of angle-closure glaucoma: prevalence, clinical types, and association with peripheral anterior chamber depth in the Egna-Neumarket Glaucoma Study. Ophthalmology 2000; 107: 998-1003.

10 Gazzard G, Friedman DS, Devereux JG, Chew P, Seah SK. A prospective ultrasound biomicroscopy evaluation of changes in anterior segment morphology after laser iridotomy in Asian eyes. Ophthalmology 2003; 110(3): 630-638.

11 Kaushik S, Jain R, Pandav SS, Gupta A. Evaluation of the anterior chamber angle in Asian Indian eyes by ultrasound biomicroscopy and gonioscopy. Indian J Ophthalmol 2006; 54(3): 159-163. 